Fluctuation and Noise Letters

Vol. 0, No. 0 (2001) 000-000

(C) World Scientific Publishing Company

\title{
FLUCTUATIONS AND NOISE IN TIME-RESOLVED LIGHT SCATTERING EXPERIMENTS: MEASURING TEMPORALLY HETEROGENEOUS DYNAMICS
}

\author{
A. Duri, P. Ballesta, L. Cipelletti \\ GDPC UMR 5581 Université Montpellier II et CNRS, P. E. Bataillon, Montpellier, France; \\ lucacip@gdpc.univ-montp2.fr \\ H. Bissig, V. Trappe \\ Department of Physics, University of Fribourg, Perolles,Fribourg, Switzerland \\ Received (received date) \\ Revised (revised date) \\ Accepted (accepted date)
}

\begin{abstract}
We use Time Resolved Correlation (TRC), a recently introduced light scattering method, to study the dynamics of a variety of jammed, or glassy, soft materials. The output of a TRC experiment is $c_{I}(t, \tau)$, the time series of the degree of correlation between the speckle patterns generated by the light scattered at time $t$ and $t+\tau$. We characterize the fluctuations of $c_{I}$ by calculating their Probability Density Function, their variance as a function of the lag $\tau$, and their time autocorrelation function. The comparison between these quantities for a Brownian sample and for jammed materials indicate unambiguously that the slow dynamics measured in soft glasses is temporally heterogeneous. The analogies with recent experimental, numerical and theoretical work on temporal heterogeneity in the glassy dynamics are briefly discussed.
\end{abstract}

Keywords: Light scattering, fluctuations, slow dynamics, glass, jamming, dynamical heterogeneity.

\section{Introduction}

Dynamic light scattering (DLS), also known as photon correlation spectroscopy, is a popular technique that provides information on the dynamics of soft condensed matter systems [1]. It is widely used in physics, chemistry, and biology to investigate systems as diverse as colloidal particle suspensions, emulsions, polymer or protein solutions, surfactant phases, and gels. In the past years, new methods and techniques have been developed that have greatly extended the range of applicability of dynamic light scattering. The advent of coherent, high brilliance X-ray sources has allowed photon correlation spectroscopy experiments to be performed using X-ray radiation [2], instead of the laser beam used for DLS. Since in a scattering experi- 
ment the wavelength of the radiation sets the length scale over which the dynamics is probed, X-ray photon correlation spectroscopy (XPCS) is a significant extension of DLS. The interpretation of both DLS and XPCS data is based on the assumption that the scattering is sufficiently weak, so that virtually all photons collected by the detector are singly scattered and the distortions induced by multiple scattering can be neglected. Diffusing Wave Spectroscopy (DWS) [3], on the contrary, allows one to study very turbid samples, for which multiple scattering dominates, thus extending the range of samples to which dynamic scattering methods can be applied.

Quite generally, in a scattering experiment the information on the dynamics is extracted from the time fluctuations of the scattered intensity, $I(t)$. The intensity is usually measured by a point detector, such as a phototube; its fluctuations are quantified by calculating the time autocorrelation function of $I(t)$, defined by $G_{2}(\tau)=\langle I(t) I(t+\tau)\rangle_{t}$, where $\langle\ldots\rangle_{t}$ denotes an average over time ${ }^{1}$. The time average is necessary to obtain sufficiently good statistics: typically, data have to be collected over a period as long as 10000 times the relaxation time of $G_{2}(\tau)$. Because of the extended time averaging, traditional scattering methods can only provide information on the average dynamics of a system, with no time resolution. The lack of time resolution, together with the need of collecting data over a long period, makes traditional scattering techniques difficult to apply to glassy —or jammed [4] — systems, for which there is currently a great interest (for a review, see for example Ref. 5). In fact, for these systems the relaxation time can be as long as several thousand seconds, thus making time averaging practically impossible. Moreover, their dynamics is often non-stationary; therefore, time-averaged correlation functions do not allow the evolution of the dynamics to be followed precisely. The introduction of the so-called multispeckle technique has addressed these issues. In a multispeckle experiment, the point detector is replaced by the 2-D array of pixels of a CCD camera [6-8]. Each CCD pixel acts as an independent detector, for which an intensity time autocorrelation function is calculated. In multispeckle experiments, the time average that is usually performed in traditional light scattering is replaced by an average over the CCD detector: $G_{2}(t, \tau)=\left\langle I_{p}(t) I_{p}(t+\tau)\right\rangle_{p}$, where the subscript $p$ refers to the CCD pixels. In most works, $G_{2}(t, \tau)$ is also averaged over a short time window [8] or over several repetitions of the experiment [9], in order to reduce the measurement "noise".

Recently, we have proposed a novel scattering method, termed Time Resolved Correlation (TRC), where valuable physical information on the dynamics are extracted precisely from this "noise", i.e. from the time fluctuations of $G_{2}(t, \tau)$ [10]. In particular, we have shown that, contrary to traditional scattering methods, TRC allows temporally heterogeneous dynamics to be discriminated from homogeneous dynamics $^{2}$. Because there is growing evidence that dynamical heterogeneity is a key feature of the slow dynamics of glassy systems, TRC appears as a very promis-

\footnotetext{
${ }^{1}$ Although in principle information on the dynamics may also be extracted from the spectral properties of the scattered light, the analysis in the temporal domain has become the standard procedure.

${ }^{2} \mathrm{As}$ it is common practice in the literature on glassy systems, in this paper we use the adjective "heterogeneous" to indicate the presence of fluctuations in the dynamics other than the inevitable noise associated with the measuring process. Note that, with this definition, both stationary and non-stationary dynamical processes may be temporally heterogeneous.
} 
ing tool for investigating the slow relaxation of these systems. In this paper, we review the principles of TRC and we propose some ways of analyzing TRC data. The paper is organized as follows: Sect. 2 briefly reviews the basic features of a scattering experiments and the way TRC data are collected. In Sect. 3 we develop the statistical analysis used to characterize the fluctuations of the TRC signal and we apply it to data collected for systems whose dynamics is homogeneous. In the following Section, the same tools are used for systems that exhibit temporal heterogeneities. Section 5 concludes the paper by summarizing the main results and briefly compares them to other experimental and numerical works on systems whose dynamics is temporally heterogeneous.

\section{Time Resolved Correlation}

The TRC method can be applied both to the single scattering geometry and to DWS experiments. In all cases the multispeckle detection is adopted: a CCD camera is used to take a time series of images of the speckle pattern, the characteristic grainy picture generated by the interference of the photons scattered by the sample.

Typical scattering setups are sketched in Fig. 1 for single (a) and multiple (b) scattering measurements; XPCS instruments are similar, the laser source being replaced by synchrotron radiation. A detailed discussion on how the speckle pattern fluctuates because of the sample dynamics is beyond the scope of this paper and can be found in Ref. 1,3. For our purposes, we just note that any change in the position of the scatterers modifies the relative phase of the scattered photons and thus the intensity pattern of the speckles generated by their interference. Using the notation of Ref. 10, we quantify the change in the speckle pattern by introducing $c_{I}(t, \tau)$, the normalized intensity correlation between speckle images at time $t$ and $t+\tau$ :

$$
c_{I}(t, \tau)=\frac{G_{2}(t, \tau)}{\left\langle I_{p}(t)\right\rangle_{p}\left\langle I_{p}(t+\tau)\right\rangle_{p}}-1=\frac{\left\langle I_{p}(t) I_{p}(t+\tau)\right\rangle_{p}}{\left\langle I_{p}(t)\right\rangle_{p}\left\langle I_{p}(t+\tau)\right\rangle_{p}}-1,
$$

where the average is over CCD pixels. The normalization in Eq. (II) guarantees that $c_{I}(t, \tau)$ is not affected by any change in the incoming beam intensity. Because of the statistical properties of the speckle pattern, $0 \leq c_{I}(t, \tau) \leq \beta$, where $\beta \leq 1$ is a prefactor that depends on the setup geometry $[1,11]$.

Figure 2 shows a 2-D grey scale representation of $c_{I}$ as a function of both time $t$ and time lag $\tau$ (data were taken in the DWS geometry for a colloidal paste [12]). White corresponds to the highest degree of correlation, $c_{I}=\beta$, while black corresponds to uncorrelated pairs of speckle patterns, $c_{I}=0$. As general trend, for any given $t c_{I}$ decreases with increasing time lag $\tau$, since the longer the lag, the greater the change of the system configuration and thus that of the speckle images. This is exemplified by the right panel that shows $c_{I}(t=3220 \mathrm{sec}, \tau)$, obtained by cutting the 2-D data along the vertical dashed line. Note that the time average of $c_{I}(t, \tau)$ yields the normalized intensity autocorrelation function, $g_{2}(\tau)-1$, usually measured in light scattering experiments ${ }^{3}$. The time fluctuations of $c_{I}$ measured at a constant $\operatorname{lag} \tau=1360 \mathrm{sec}$, obtained from a cut of the 2-D data along the horizontal solid line, are shown in the top panel. Sharp drops of correlation that depart from the typical

\footnotetext{
${ }^{3}$ In traditional light scattering measurements, the average is exclusively over time when using a point detector, over both pixels and time when using a CCD camera.
} 
Fluctuations and noise in time-resolved light scattering
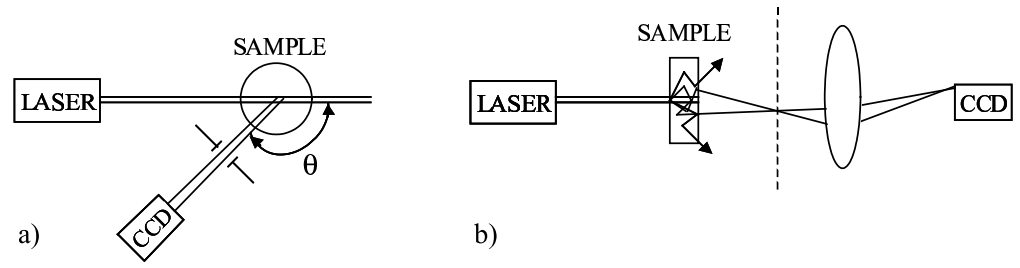

b)

Fig 1. Schematic representation of a) a single scattering apparatus, b) a multiple scattering (DWS) setup in the transmission geometry. In a), $\theta$ is the scattering angle, selected by the diaphragm in front of the CCD. The lens in b) conjugates the plane indicated by the dashed line with the CCD chip. In both cases, the CCD camera is used to record the speckle pattern generated by the interference of the scattered photons. 


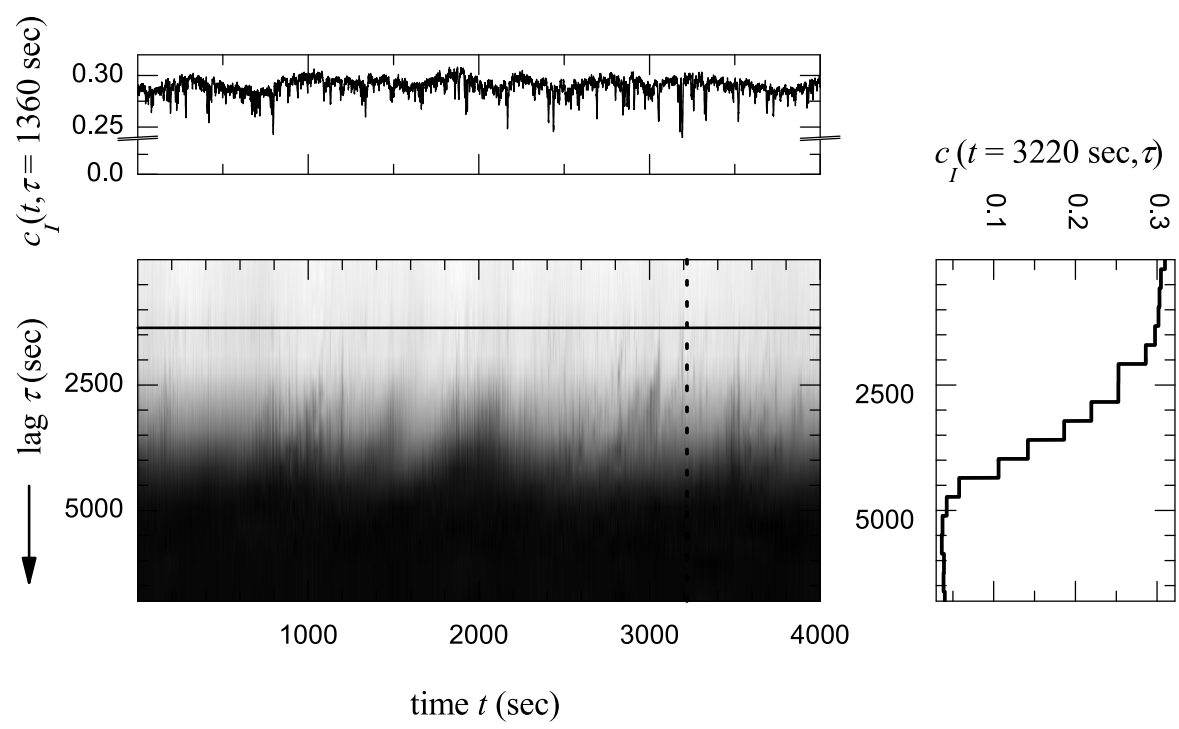

Fig 2. Bottom left panel: 2-D grey scale representation of $c_{I}(t, \tau)$, the degree of correlation between speckle patterns recorded at time $t$ and $t+\tau$. White is the highest correlation, black the lowest. The sample is a concentrated colloidal paste. Right panel: two-time intensity autocorrelation function obtained by cutting $c_{I}$ along the vertical dashed line. Top panel: temporal fluctuations of $c_{I}$ at a fixed lag time, obtained from a cut along the horizontal solid line. Note the sharp drops of correlation, indicative of sudden, temporally localized rearrangements. 
fluctuations of the signal are clearly visible. These drops suggest that occasionally the change in the speckle pattern, and thus in sample configuration, is much larger than average: they are therefore indicative of a temporally heterogeneous dynamics. In the rest of the paper, we will focus on the statistical analysis of the fluctuations of $c_{I}(t, \tau)$ at fixed lag $\tau$, first for samples that exhibit homogeneous dynamics, and then for systems whose dynamics is temporally heterogeneous.

\section{Statistical Analysis of TRC Data for temporally homogeneous dy- namics}

In order to better appreciate the signature of heterogeneous dynamics in TRC data, we first characterize the statistics of the fluctuations of $c_{I}(t, \tau)$ in the case of temporally homogeneous dynamics. The data presented in this Section are taken in the single scattering geometry, at a scattering angle $\theta=90 \mathrm{deg}^{4}$. The sample is a diluted suspensions of particles undergoing Brownian motion: polystyrene spheres of radius $r=530 \mathrm{~nm}$ were suspended at a volume fraction $\phi=3.7 \times 10^{-5}$ in almost pure glycerol (approximately 99.9/0.001 w/w glycerol/water). The sample temperature was controlled and fixed at $T=15 \pm 0.1^{\circ} \mathrm{C}$.

Figure 3a) shows the time-averaged intensity autocorrelation function $g_{2}(\tau)-1$ obtained from the TRC data for the Brownian particles. The data are well fitted by an exponential decay $\beta \exp \left(-\tau / \tau_{s}\right)$ (line in the figure), as expected for a dilute suspension of monodisperse spheres [1], with $\tau_{s}=2.35 \mathrm{sec}$. The right panel shows the corresponding $c_{I}(t, \tau)$ as a function of time $t$ for four time lags, ranging from $\tau / \tau_{s}=0.21$ to $\tau / \tau_{s}=6.38$. For Brownian particles, we expect the dynamics to be temporally homogeneous. Accordingly, the loss of correlation between pairs of speckle images separated by a given time lag $\tau$ should not depend on the time the first image of the pair is taken. In other words, for a fixed lag $\tau, c_{I}(t, \tau)$ should be constant. This is indeed the case, except for the small fluctuations visible in in the right panel, which we attribute to the CCD noise and the finite number of pixels, and therefore independent speckles, over which $c_{I}$ is averaged. In the following we shall refer to both sources of noise as to the "measurement noise".

In order to verify this hypothesis, we analyze in some detail the temporal fluctuations of $c_{I}$. Figure 4 shows the Probability Density Function (PDF) of $c_{I}$ for the same time lags as in Fig. 3 right panel. At all lags, the data are well fitted by a Gaussian distribution, as shown by the lines. This behavior is consistent with the hypothesis that the fluctuations are due to the measurement noise. Note that the width of the distributions decreases for increasing lag $\tau$, together with the mean value $g_{2}(\tau)-1=\left\langle c_{I}\right\rangle_{t}$. To better investigate the lag dependence of the amplitude of the fluctuations, we plot in the left panel of Fig. [5 the variance of $c_{I}, \operatorname{var}\left(c_{I}\right)$, as a function of $\tau$ normalized by the mean relaxation time $\tau_{s}$. At the smaller lags, the decay of the amplitude of the fluctuations closely follows that of $g_{2}(\tau)-1$, whereas for $\tau>\tau_{s} \operatorname{var}\left(c_{I}\right)$ saturates to a plateau. This behavior suggests the following form for the variance of $c_{I}: \operatorname{var}\left(c_{I}\right)=A+B\left(\left\langle c_{I}\right\rangle_{t}+1\right)^{2}$, where the second term of the r.h.s. represents a noise contribution proportional to $G_{2}(t, \tau) /\left\langle I_{p}(t)\right\rangle_{p}\left\langle I_{p}(t+\tau)\right\rangle_{p}$, the signal that is measured in a TRC experiment. The first term is the noise of $c_{I}$ when all correlation between speckle images is lost, i.e. the fluctuations of the

${ }^{4}$ Similar results are obtained in DWS 
A. Duri, P. Ballesta, L. Cipelletti, H. Bissig and V. Trappe

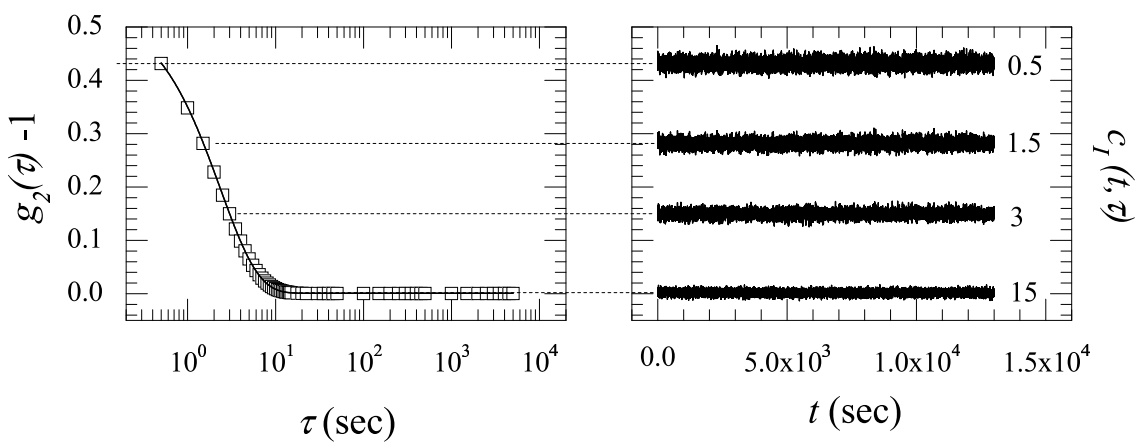

Fig 3. For a dilute suspension of Brownian particles are shown: left panel: the intensity autocorrelation function $g_{2}(\tau)-1$; right panel: $c_{I}(t, \tau)$ as a function of $t$ for four time lags $\tau$. The correlation function shown in the left panel was obtained by time-averaging the TRC data plotted in the right panel (for clarity, not all the time lags are shown in the right panel). 
Fluctuations and noise in time-resolved light scattering

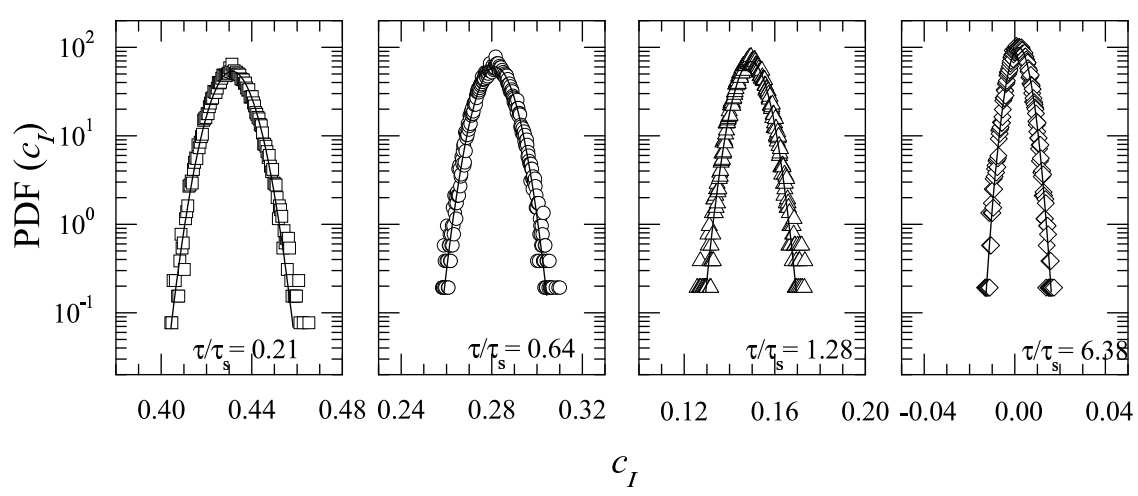

Fig 4. Probability Density Function of the temporal fluctuations of $c_{I}(t, \tau)$, for the data shown in Fig. 3 b) (symbols: from left to right, $\tau=0.5,1.5,3$, and $15 \mathrm{sec}$ ). The lines are Gaussian fits to the data. Note that width oh the PDF's - and thus the standard deviation of the fluctuationsdecreases at larger lags, together with the mean of $c_{I}$. 
base line for $\tau \rightarrow \infty$. As it will be discussed in more detail in reference to Fig. [6] this contribution is mainly due to the finite number of pixels. The right panel of Fig. [5 shows $\operatorname{var}\left(c_{I}\right)$ vs $\left(\left\langle c_{I}\right\rangle_{t}+1\right)^{2}$ (symbols). The data are in good agreement with the linear behavior $\operatorname{var}\left(c_{I}\right)=A+B\left(\left\langle c_{I}\right\rangle_{t}+1\right)^{2}$ introduced above, except for the downturn visible at the smallest values of $\left\langle c_{I}\right\rangle_{t}$ (the straight line is a linear fit for $\left.\left(\left\langle c_{I}\right\rangle_{t}+1\right)^{2}>1.3\right)$. The origin of this deviation most likely lies in correlations between $G_{2}(t, \tau)$ and $\left\langle I_{p}(t)\right\rangle_{p}$. We are currently investigating the origin of this issue.

The measurement noise is expected to increase as the number of pixels over which the intensity correlation is averaged, $n_{p i x}$, decreases. More precisely, the variance of the CCD electronic (or dark) noise and read-out noise should scale as $n_{\text {pix }}^{-1}$, because of the Central Limit Theorem. Similarly, even in the absence of CCD noise, all pixel-averaged quantities in Eq. (1) will fluctuate as the speckle pattern is renewed over time, because $n_{p i x}$ is finite [11]. By invoking again the Central Limit Theorem, we expect the variance of these fluctuations to exhibit the same $n_{\text {pix }}^{-1}$ scaling as those due to the CCD noise. Therefore, the overall variance of the measurement noise should decay with $n_{p i x}$ as a power law with exponent -1 . The left panel of Fig. [6] shows that indeed this behavior holds for all $\tau$, both smaller and larger than the mean relaxation time $\tau_{s}$, thus further supporting the hypothesis that the fluctuations of $c_{I}$ for temporally homogeneous systems are due to the measurement noise.

The PDF and the variance of $c_{I}$ that we have so far introduced are not sensitive to the detailed way $c_{I}$ fluctuates over time, but only to its probability to assume a given value. To investigate the time behavior of the fluctuations, we calculate the time autocorrelation function of $c_{I}(t, \tau)$, defined as

$$
\operatorname{Corr}\left(c_{I, \tau}\right)(\Delta t)=\frac{\left\langle\left(c_{I}(t, \tau)-\left\langle c_{I}\right\rangle_{t}\right)\left(c_{I}(t+\Delta t, \tau)-\left\langle c_{I}\right\rangle_{t}\right)\right\rangle_{t}}{\left\langle\left(c_{I}(t, \tau)-\left\langle c_{I}\right\rangle_{t}\right)^{2}\right\rangle_{t}},
$$

where we have added the index $\tau$ to designate the time lag for which $c_{I}$ is calculated. Note that with the above definition $\operatorname{Corr}\left(c_{I, \tau}\right)(0)=1$ and $\operatorname{Corr}\left(c_{I, \tau}\right)(\Delta t)=0$ for uncorrelated signals (e.g. for $\Delta t \rightarrow \infty$ ). The quantity introduced in Eq. (2) is a fourth-order time correlation function, since $c_{I}$ itself is a time correlation function. In order to avoid any confusion, in the following we shall refer to the argument $\Delta t$ of $\operatorname{Corr}\left(c_{I, \tau}\right)$ as to the time delay, as opposed to the time lag $\tau$ for $c_{I}$. In the right panel of Fig. [6 $\operatorname{Corr}\left(c_{I, \tau}\right)(\Delta t)$ is plotted as a function of $\Delta t$ normalized by the relaxation time $\tau_{s}$, for the same four time lags as in the previous figures of this section. As it can be seen, $\operatorname{Corr}\left(c_{I, \tau}\right)(\Delta t)$ decays to 0 on a time scale comparable to the relaxation time of the sample, $\tau_{s}$. Indeed, the data can be fit by an exponential relaxation, whose characteristic time varies between $0.21 \tau_{s}$ and $0.31 \tau_{s}$ (for clarity, only the fit for the data collected at $\tau=1.5 \mathrm{sec}$ is shown, as a solid line, in the figure). At first sight this behavior may seem in contrast with the assumption that here the fluctuations of $c_{I}$ are due to the measurement noise, which is expected to be uncorrelated. Indeed, this is the case for the CCD noise, as we tested by taking a series of dark images. However, we recall that the fluctuations of the quantities $\left\langle I_{p}(t) I_{p}(t+\tau)\right\rangle_{p}$ and $\left\langle I_{p}\right\rangle_{p}$ used to compute $c_{I}$ are due not only to the CCD noise, but also to the finite number of speckles that are sampled when averaging over the CCD pixels. This latter contribution to the noise of $c_{I}(t, \tau)$ is correlated over a time scale comparable to $\tau_{s}$, for all time lags $\tau$. To demonstrate it, let us consider 
Fluctuations and noise in time-resolved light scattering
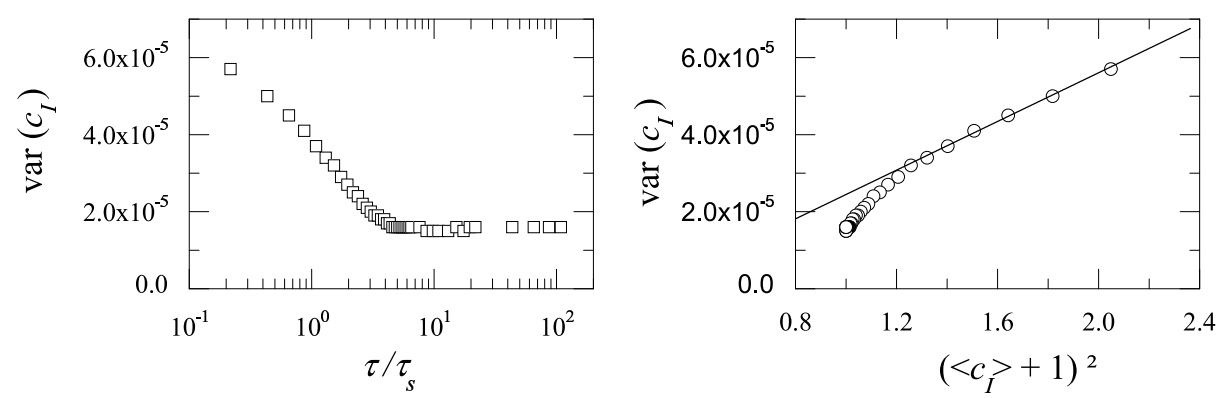

Fig 5. Left: lag dependence of the variance of the temporal fluctuations of $c_{I}$ for a suspensions of Brownian particles. Right: same data plotted parametrically $v s$ the squared mean of the TRC signal, $\left(\left\langle c_{I}\right\rangle_{t}+1\right)^{2}$. Except for the lowest values of $\left(\left\langle c_{I}\right\rangle_{t}+1\right)^{2}$, the data follow a linear behavior, as shown by the straight line. 
A. Duri, P. Ballesta, L. Cipelletti, H. Bissig and V. Trappe
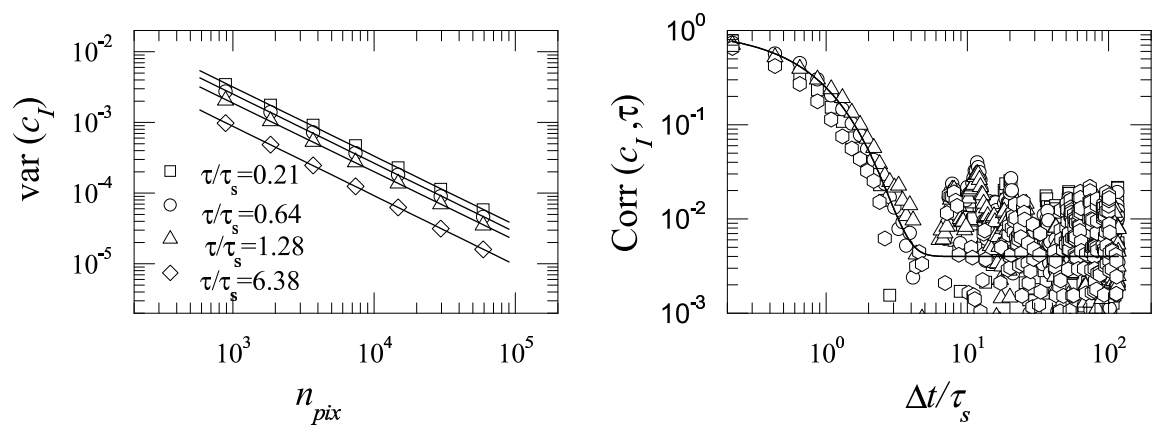

Fig 6. For a suspension of Brownian particles are shown: Left panel: double logarithmic plot of the variance of the temporal fluctuations of $c_{I}$, as a function of the number of processed pixels, and for various time lags. For all $\tau$, the data are well fitted by a power law decay with exponent $-0.98 \pm 0.01$, very close to what is expected for the measurement noise because of the Central Limit Theorem. Right panel: time autocorrelation function of the fluctuations of $c_{I}$, for various lags (symbols). The line is an exponential fit to the data for $\tau=1.5 \mathrm{sec}$ (the fit for the other values of $\tau$ are omitted for clarity). The correlation in the fluctuations stems from the finite number of pixels over which $c_{I}$ is averaged, as discussed in the text. 
as an example the fluctuations of $c_{I}(t, \tau)$ for $\tau \gg \tau_{s}$, so that $\left\langle c_{I}(t, \tau)\right\rangle_{t}=0$. A measurement of $c_{I}$ at a given time $t$ will yield a value $\epsilon$ that is in general slightly different from zero, because $c_{I}$ is averaged over a finite number of pixels. If the measurement is repeated at a later time $t+\Delta t$, with $\Delta t \ll \tau_{s}$, the outcome will be essentially the same, since the system configuration is almost frozen on a time scale $\Delta t \ll \tau_{s}$ and thus the speckle patterns recorded at time $t+\Delta t$ and $t+\tau+\Delta t$ are almost unchanged with respect to those recorded at time $t$ and $t+\tau$, respectively. Therefore, $c_{I}(t+\Delta t, \tau)$ is highly correlated to $c_{I}(t, \tau)$. As the delay is increased, the evolution of the speckle images during $\Delta t$ will become increasingly relevant and $c_{I}(t+\Delta t, \tau)$ and $c_{I}(t, \tau)$ will become less and less correlated. Since the speckle fields are renewed completely after a few $\tau_{s}$, one expects $\operatorname{Corr}\left(c_{I, \tau}\right)(\Delta t)$ to decay with a characteristic time close to $\tau_{s}$, as observed in the right panel of Fig. [6] (note that similar arguments can be invoked for all $\tau$ ).

\section{Statistical Analysis of TRC data for temporally heterogeneous dy- namics}

We now turn to TRC data collected for jammed systems and show that the statistical properties of $c_{I}$ are very different from those reported in the previous section for dilute Brownian particles. Although we will focus in turn on different systems, such as a micellar polycrystal, the colloidal paste cited above, and a shaving cream foam, we stress that most of the features that will be shown appear to be quite general. As we shall discuss in the following, these differences indicate that the slow dynamics of jammed systems is temporally heterogeneous.

Figure 7 shows TRC data collected in the single scattering geometry $(\theta=45 \mathrm{deg})$ for the slow dynamics of the network of defects of a micellar polycrystal (more details on the sample and its average dynamics can be found in Ref. 13 and the references therein). For this system, the time-averaged intensity autocorrelation function is a "compressed" exponential: $g_{2}(\tau)-1=\beta \exp \left[-\left(\tau / \tau_{s}\right)^{p}\right]$, with $\tau_{s}=3160 \mathrm{sec}$ and $p \approx 1$.7. Similarly to the case of the colloidal paste data shown in the upper panel of Fig. 2. the fluctuations of $c_{I}$ are very different from those measured for a Brownian suspension (compare to Fig. 3. right panel). As discussed previously, the large drops of $c_{I}(t, \tau)$ below its mean value indicate that occasionally the sample undergoes a rearrangement that is much larger than the typical change in configuration over a time $\tau$. Therefore, the TRC data unambiguously show that the dynamics of both the colloidal paste and the micellar polycrystal is intermittent. The PDF of $c_{I}(t, \tau=750 \mathrm{sec})$ is shown in the right panel. Because of the drops of the signal visible in the left panel, the PDF severely deviates from the Gaussian shape observed for the Brownian sample (compare to Fig. 4) and is strongly skewed to the left, the left tail being close to an exponential decay. Interestingly, the shape of the PDF depends on the time lag at which $c_{I}$ is measured. Without discussing the details of this dependence, we remark that for $\tau \gg \tau_{s}$ the PDF recovers a Gaussian shape (data not shown). This behavior can be understood by assuming that the average time between rearrangements is much shorter than the mean relaxation time $\tau_{s}$. Accordingly, a large number of rearrangements occur between any two images taken at a lag $\tau \gg \tau_{s}$ and the PDF is Gaussian because of the Central Limit Theorem. In other words, on these time scales the dynamics is due to such a large 
A. Duri, P. Ballesta, L. Cipelletti, H. Bissig and V. Trappe
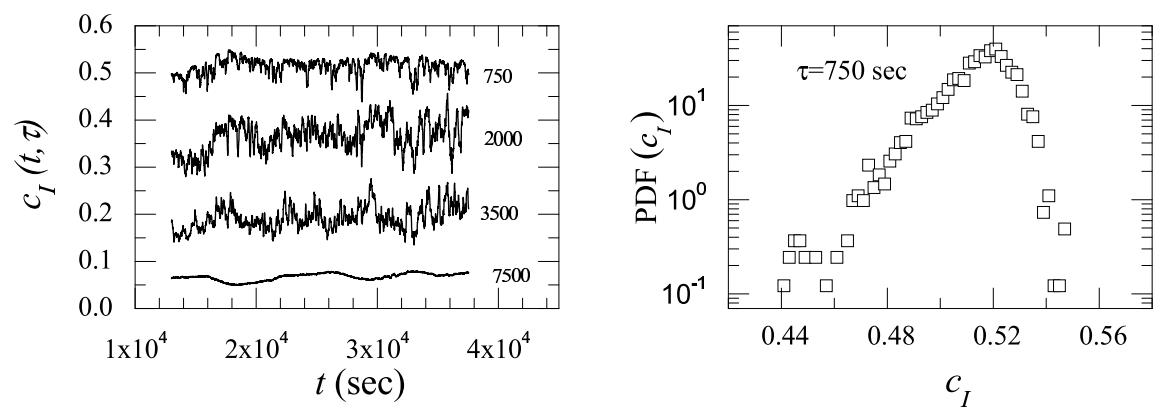

Fig 7. For a micellar polycrystal are shown: Left panel: time variation of $c_{I}(t, \tau)$ for several lags (the curves are labelled by the normalized lag $\tau / \tau_{s}$ ). The relaxation time of $g_{2}-1$ is $\tau_{s}=3158.5$ sec. Right panel: for the same data, PDF of $c_{I}$ for $\tau / \tau_{s}=0.237$. Note the highly skewed shape of the PDF, due to the marked drops of correlation visible in the left panel. For the longest $\tau$, a Gaussian shape of the PDF is recovered (not shown in the figure). 
number of individual rearrangement events that it can be regarded as continuous, much as for a Brownian suspension the dynamics is continuous because it arises from the motion of a very large number of particles, even though each individual particle moves in a jerky fashion.

We continue the comparison between the statistical properties of TRC data for jammed systems and those for fluid systems by contrasting the $\tau$ dependence of the variance of $c_{I}$ for the colloidal paste introduced in Sect. 2 to that for the diluted Brownian suspension, shown previously in Fig. [5 As can be seen in Fig. 8 for the colloidal paste $\operatorname{var}\left(c_{I}\right)$ is peaked around $\tau \approx \tau_{s}$ (for this sample, $g_{2}(\tau)-$ $1 \approx \beta \exp \left(-\tau / \tau_{s}\right)$, with $\left.\tau_{s}=1580 \mathrm{sec}\right)$, instead of decaying with $\tau$, as for the Brownian particles. We stress that the peaked shape is conserved even when plotting parametrically $\operatorname{var}\left(c_{I}\right)$ vs $\left(\left\langle c_{I}\right\rangle_{t}+1\right)^{2}$ (data not shown), contrary to the case of the Brownian suspension (see Fig. [5 right panel). Moreover, the peak value of the variance is 2 orders of magnitude larger than the measurement noise at large $\tau$, whereas for the Brownian sample the difference between the smallest and largest value of $\operatorname{var}\left(c_{I}\right)$ is only about a factor of 3 . Therefore, both the presence of a peak and the larger amplitude of the fluctuations clearly differentiate the dynamics of the pastes from that of the Brownian particles, even though the shape of the time-averaged correlation function is the same for both systems, i.e. an exponential decay. The presence of a peak in $\operatorname{var}\left(c_{I}\right)$ can be understood, at least qualitatively, by assuming that the characteristic time of the relaxation of $c_{I}$ fluctuates with time, $\tau_{s}=\tau_{s}(t)$, rather than being constant. To illustrate schematically this point, we show in the right panel of Fig. 8 two exponential decays with slightly different relaxation times (in arbitrary units), $\tau_{s}=1$ and 1.5 , respectively. These two curves are meant to be representative of the typical behavior of $c_{I}$ vs $\tau$ measured at two different times $t_{1}$ and $t_{2}$. As indicated by the arrows, the resulting change in correlation at a given lag is maximum for $\tau \approx \tau_{s}$ and decreases both at small and large lags, in qualitative agreement with the curve shown in the left panel. For our jammed systems, the physical origin of the fluctuations of $\tau_{s}$ most likely lies in the fluctuation of the number, or the size, of the regions that rearranged by each event. More work is currently being carried on to explore quantitatively this concept.

To investigate how the fourth-order correlation function $\operatorname{Corr}\left(c_{I, \tau}\right)(\Delta t)$ behaves when the dynamics is temporally heterogeneous, we analyze TRC data collected in the DWS transmission geometry for a shaving cream foam. Previous work by Durian et al. has shown that the dynamics of a foam is intermittent, due to bubble rearrangements that are localized both in time and in space [14]. Therefore, the foam represents an ideal system for testing the TRC method. In our experiment, we find that the time-averaged correlation function $g_{2}-1$ is well fitted by an exponential decay with characteristic time $\tau_{s}=0.29 \mathrm{sec}$, in agreement with Ref. 14 . The left panel of Fig. 9 shows $\operatorname{Corr}\left(c_{I, \tau}\right)$ vs. the normalized delay $\Delta t / \tau_{s}$ for various lags $\tau$. In sharp contrast with the behavior of the fourth-order correlation for the Brownian sample (see Fig. [6 right panel), for the foam $\operatorname{Corr}\left(c_{I, \tau}\right)$ strongly depends on the lag at which $c_{I}$ is calculated, the decay time of $\operatorname{Corr}\left(c_{I, \tau}\right)$ increasing with $\tau$. To characterize this dependence, for each $\tau$ we extract from the initial relaxation of $\operatorname{Corr}\left(c_{I, \tau}\right)$ a (normalized) characteristic time, $\Gamma_{90}$, which we define 
A. Duri, P. Ballesta, L. Cipelletti, H. Bissig and V. Trappe
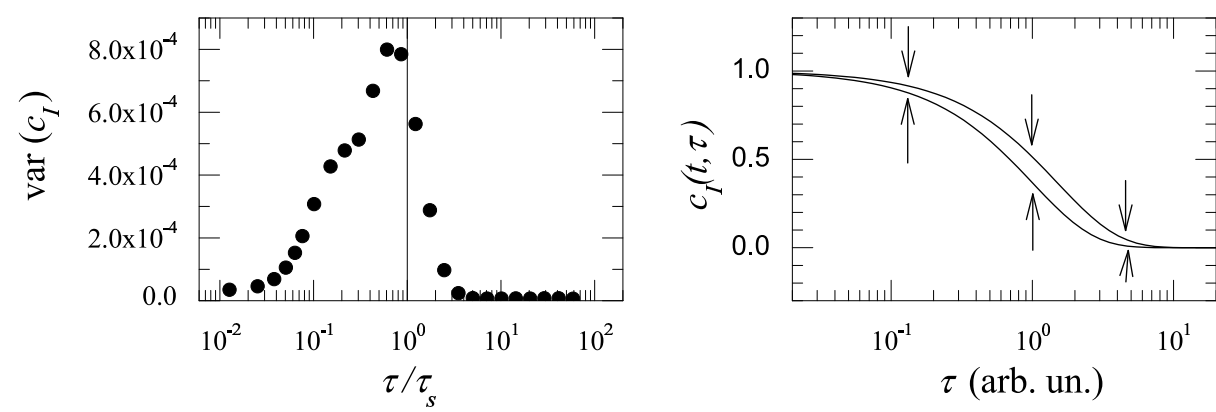

Fig 8. Left panel: $\tau$ dependence of the variance of $c_{I}$ for a dense colloidal paste. Data are taken in the DWS transmission geometry. Note the peak of $\operatorname{var}\left(c_{I}\right)$ for $\tau \approx \tau_{s}$ and the large value of the fluctuations at the peak, compared to that for $\tau \gg \tau_{s}$. Right panel: schematic representation of the impact of a fluctuation of the relaxation time $\tau_{s}$ on the value of $c_{I}$. As shown by the arrows, the change of $c_{I}$ at a fixed lag is maximum for $\tau \approx \tau_{s}=1$. 
Fluctuations and noise in time-resolved light scattering
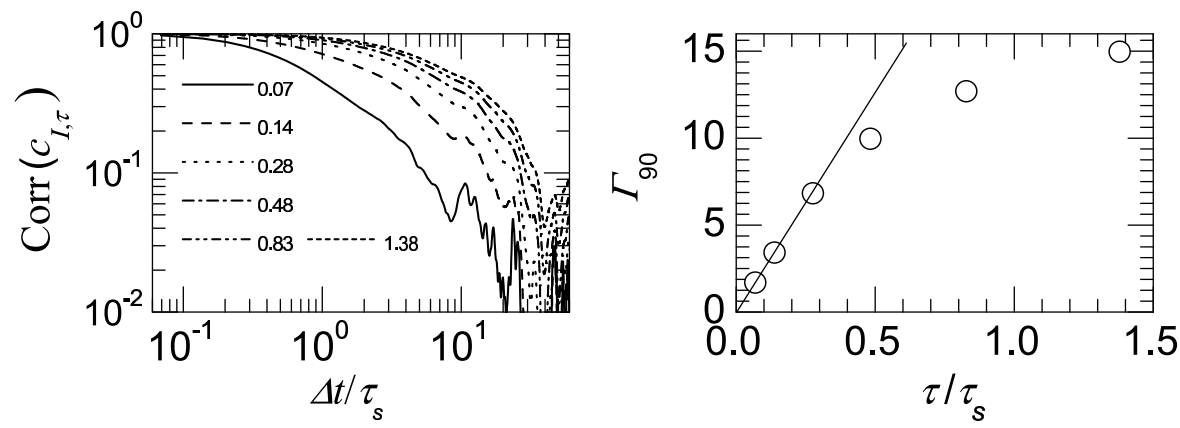

Fig 9. For DWS measurements on a shaving cream foam are shown: Left panel: the time autocorrelation of $c_{I}$, for various lags $\tau$, as a function of the normalized delay $\Delta t / \tau_{s}$. The curves are labelled by $\tau / \tau_{s}$. Contrary to the case of Brownian particles, there is a strong $\tau$ dependence of the decay rate of $\operatorname{Corr}\left(c_{I, \tau}\right)(\Delta t)$. Right panel: $\tau$ dependence of the relaxation time of the curves shown in the left panel (symbols, see text for details). For the shortest lags, $\Gamma_{90}$ is proportional to $\tau$, as indicated by the straight line. 
as the (normalized) delay for which $\operatorname{Corr}\left(c_{I, \tau}\right)=C_{t h} \equiv 0.90{ }^{5}$. We plot $\Gamma_{90}$ as a function of $\tau$ in the right panel of Fig. 9] as it can be seen, for small lags $\Gamma_{90}$ is proportional to $\tau$, whereas it deviates from this linear trend as $\tau$ approaches the relaxation time of $g_{2}-1$. Therefore, for the foam the fluctuations of $c_{I}(t, \tau)$ are correlated over a time scale proportional to $\tau$, at least as long as the time lag is not too large.

In order to explain this behavior, we represent schematically the evolution of a system whose dynamics is intermittent in Fig. 10. The rectangles at different grey levels represent different configurations of the system: the evolution occurs via discrete "jumps" - or rearrangements - from a frozen configuration to the next one, rather than through a continuous change. The life time of the configurations is assumed to vary randomly. The degree of correlation between pairs of speckle images drops whenever the two images are taken for different configurations, while it stays high within the same configuration (see the sketch of $c_{I}$ below the time axis in Fig. 10] for two values of $\tau$ ). This implies that the dips of $c_{I}$ have a width equal to the time lag $\tau$, and that their raising edge is aligned with the change in configuration, thus allowing one to identify precisely the time at which the rearrangement event occurred. Accordingly, for any given $\tau$, the TRC signal consist of a random sequence of downward pulses of width $\tau$. The autocorrelation of such a signal decays over a time scale of the order of the width of the pulses, i.e. over a time of order $\tau$. Therefore, this rather crude picture provides some explanation to the proportionality between the decay time of $\operatorname{Corr}\left(c_{I, \tau}\right)$ and $\tau$ that is observed, at small $\tau$, for the foam. Note that as the lag increases the likelihood of two or more rearrangements to occur during a time $\tau$ becomes increasingly relevant. These multiple rearrangements would alter the shape of the dips of $c_{I}$, thus reducing the decay time of $\operatorname{Corr}\left(c_{I, \tau}\right)$ with respect to what is expected by extrapolating the small $\tau$ regime, as observed in Fig. 9 (right panel). Of course, a more realistic description of the dynamics should take into account the possibility that the rearrangement be more gradual and the dynamics within a given configuration not completely frozen. Moreover, in a macroscopic scattering volume many rearrangements are likely to occur at any given time, so that in practice $\tau$ would be always longer than the mean life of the configuration. This being the case, a more appropriate approach would be to relate the fluctuations of $c_{I}(t, \tau)$ to those of the number of rearrangements during a time $\tau$, rather to the occurrence of single events. We are currently exploring this scenario.

\section{Conclusions}

We have shown that, contrary to traditional light scattering techniques, TRC experiments have the capability of discriminating between continuous and temporally heterogeneous dynamics. Among the numerous ways one could analyze the TRC time series, three methods seem particularly interesting: the characterization of the PDF of the fluctuations of $c_{I}$, the study of the $\tau$ dependence of the width of the distributions, $\operatorname{var}\left(c_{I, \tau}\right)$, and the investigation of the temporal correlations via $\operatorname{Corr}\left(c_{I, \tau}\right)$. Other possible approaches may include a wavelet analysis of $c_{I}$ and the investigation of the fluctuations in the framework of chaos theory. Quite generally,

\footnotetext{
${ }^{5}$ Similar results are obtained for different $c_{t h}$, provided that $C_{t h}>0.7$.
} 
Fluctuations and noise in time-resolved light scattering

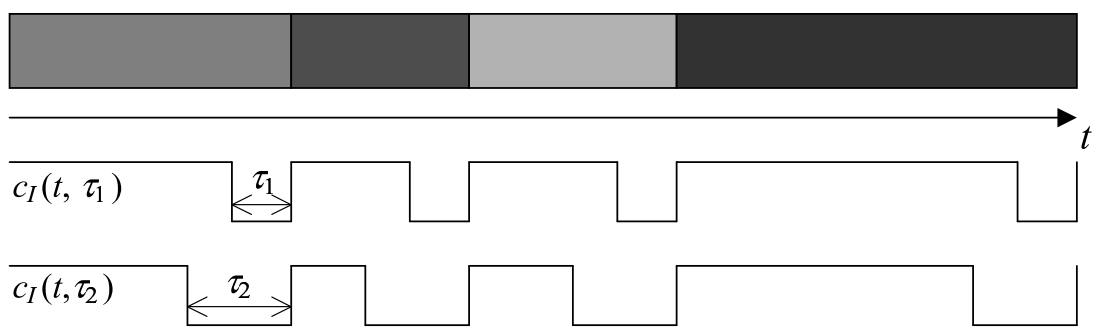

Fig 10. Schematic representation of the evolution of a system, such a foam, whose dynamics is intermittent: each rectangle represents a (slightly) different configuration of the system. The time evolution of $c_{I}$ for two values of the lag, $\tau_{1}$ and $\tau_{2}$, is shown below the time axis: $c_{I}$ drops whenever the two speckle images that are correlated are taken for different sample configurations. Note that the width of the dips is equal to the time lag. 
TRC measurements indicate that the slow dynamics of soft jammed materials is temporally heterogeneous, in striking analogy with recent numerical and experimental work on hard condensed matter glasses (for a review, see Refs. 15,16). We conclude by a brief, and partial, overview of some of the recent work related to the investigation presented in this paper, either because of the techniques that are used or in view of the results that are obtained. Dixon and Durian have introduced a method termed Speckle Visibility Spectroscopy (SVS), where the fast dynamics of a multiply scattering sample is studied by measuring with a CCD the time-dependent contrast of the speckle pattern, defined by $\left\langle I_{p}(t)^{2}\right\rangle_{p} /\left\langle I_{p}(t)\right\rangle_{p}^{2}-1$ [17]. Note that, in our notation, this quantity corresponds to $c_{I}(t, \tau=0)$. If the speckles fluctuate on a time scale shorter than or comparable to the CCD exposure time, the image will be blurred and the contrast reduced. Therefore, SVS provides information on the dynamics on a time scale comparable to the exposure time (typically from a fraction of msec to a fraction of sec), while in the TRC method the shortest time scale is set by the CCD acquisition rate (typically a few tens of images per second) and the longest one can be as large as several hours. Other similarities exist between the TRC and the work by Lemieux and Durian, who use fourth-order intensity correlation functions to measure intermittent dynamics $[18,19]$. Although the quantity $\operatorname{Corr}\left(c_{I, \tau}\right)$ introduced here is also a fourth-order intensity correlation function, we note that in Refs. 18,19 a point detector is used and no pixel average is performed, as opposed to the way $c_{I}$ is calculated. An extended time average is thus required to reduce the measurement noise; contrary to TRC, no time resolution can be obtained: it is possible to access some of the average statistical properties of the fluctuations, not their detailed temporal behavior. An interesting parallel can be made between the work presented here and the investigation of temporally heterogeneous dynamics in 2 -D electron systems $[20,21]$. Following a method originally designed by Weissman in the context of spin glasses [22], a time series of the fluctuations of the resistance (or the conductivity) is divided into shorter segments. For each segment, the power spectrum of the fluctuations is calculated. Finally, the so-called second spectrum is obtained by calculating the power spectrum of the time series of spectra calculated for the individual data segments. Because of the relationship between power spectrum and autocorrelation function of a signal, the fourth-order autocorrelation function $\operatorname{Corr}\left(c_{I, \tau}\right)$ calculated here for TRC represents the time-domain analogue of the analysis proposed in Ref. 22. The PDF of the fluctuations of the dynamics of a polymeric glass, as obtained from dielectric measurements, has been reported by Buisson et al. $[23,24]$. Skewed, non-Gaussian PDFs are observed, similar to that reported in Fig. 7 Non-Gaussian distributions are also observed in simulations of spin glasses, where the dynamics was averaged over subregions instead of over the whole simulation box $[25,26]$. Interestingly, for both our data (Fig. 17) and the simulations on spin glasses, the shape of the PDF is very close to the universal non-Gaussian PDF proposed for the fluctuations in highly correlated systems [27]. In our case, however, we recall that the shape of the PDF depends on the lag $\tau$ and that for $\tau \gg \tau_{s}$ a Gaussian distribution is recovered. Several recent works have focussed on the variance of the fluctuations of the dynamics in glassy systems. In particular, Lačević et al. [28] introduce in simulations of glass formers a time-dependent "order parameter" $Q(t)$ that compares the system configuration at two times separated by $t$ and then calculate the (normalized) variance of $Q(t), \chi_{4}(t)$. These quantities 
Fluctuations and noise in time-resolved light scattering

are analogous to our $c_{I}$ and $\operatorname{var}\left(c_{I}\right)$, respectively, since $c_{I}$ measures the degree of overlap between sample configurations separated by a lag $\tau$. Indeed, $\chi_{4}(t)$ is found to be peaked around the characteristic time of the final relaxation of $Q(t)$, much as, in our experiments, $\operatorname{var}\left(c_{I}\right)$ is peaked around $\tau_{s}$. As a final remark, we point out that in most of the recent work on heterogeneous dynamics in glassy systems, a key role is played by spatial heterogeneity and spatial correlations between the rearrangements. In our experiments, each CCD pixel is flooded by light issued by the whole scattering volume (see Fig. 11). Accordingly, any information on the spatial localization of the rearrangements is lost and only global temporal heterogeneity can be detected. We are currently working on a modified TRC scheme to overcome this limitation and achieve space- and time-resolved scattering measurements.

\section{Acknowledgements}

A. D., P. B., and L. C. acknowledge CNES (grant no. 03/CNES/4800000123), the French Ministry of Research (ACI "Jeunes chercheuses, jeunes chercheurs" JC2076) and the European Community (grant MRT-CT-2003-504712) for supporting this work. H. B. and V. T. were supported by the Swiss National Fond (grant 2065019.01). The collaboration between Montpellier and Fribourg groups is supported by CNRS (PICS no. 2410). It is a pleasure to thank E. Pitard and L. Berthier for many useful discussions and D. Popovic for bringing to our attention the work by M. B. Weissman.

\section{References}

[1] B. J. Berne and R. Pecora, Dynamic Light Scattering, Wiley, New York (1976).

[2] S. B. Dierker, R. Pindak, R. M. Fleming, I. K. Robinson, and L. Berman, "X-ray photon-correlation spectroscopy study of brownian-motion of gold colloids in glycerol," Physical Review Letters 75(3) (1995) 449-452.

[3] D. A. Weitz and D. J. Pine, "Diffusing-wave spectroscopy," in Dynamic Light scattering, W. Brown, ed., Clarendon Press, Oxford (1993) 652-720.

[4] A. Liu and S. Nagel, "Jamming is not just cool anymore," Nature 396 (1998) 21.

[5] L. Cipelletti and L. Ramos, "Slow dynamics in glasses, gels, and foams," Curr. Opin. Colloid Interface Sci. 7 (2002) 228-234.

[6] A. P. Y. Wong and P. Wiltzius, "Dynamic light-scattering with a ccd camera," Review of Scientific Instruments 64(9) (1993) 2547-2549.

[7] S. Kirsch, V. Frenz, W. Schartl, E. Bartsch, and H. Sillescu, "Multispeckle autocorrelation spectroscopy and its application to the investigation of ultraslow dynamical processes," Journal of Chemical Physics 104(4) (1996) 1758-1761.

[8] L. Cipelletti and D. Weitz, "Ultralow-angle dynamic light scattering with a charge coupled device camera based multispeckle, multitau correlator," Rev. Sci. Instrum. 70(8) (1999) 3214.

[9] V. Viasnoff and F. Lequeux, "Rejuvenation and overaging in a colloidal glass under shear," Physical Review Letters 89 (2002) 065701.

[10] L. Cipelletti, H. Bissig, V. Trappe, P. Ballesta, and S. Mazoyer, "Time resolved correlation: a new tool for studying temporally heterogeneous dynamics," J. Phys.: Condens. Matter 15 (2003) S257-S262. 
[11] J. W. Goodman, "Statistical properties of laser speckles patterns," in Laser speckles and related phenomena, J. C. Dainty, ed., Topics in Applied Physics 9, SpringerVerlag, Berlin (1975) 9-77.

[12] P. Ballesta, C. Ligoure, and L. Cipelletti, "Temporal heterogeneity of the slow dynamics of a colloidal paste," to appear in the AIP Conference Proceedings (2004).

[13] L. Cipelletti, L. Ramos, S. Manley, E. Pitard, D. A. Weitz, E. E. Pashkovski, and M. Johansson, "Universal non-diffusive slow dynamics in aging soft matter," Faraday Discuss. 123 (2003) 237-251.

[14] D. J. Durian, D. J. Pine, and D. A. Weitz, "Multiple light-scattering probes of foam structure and dynamics," Science 252(5006) (1991) 686-688.

[15] S. C. Glotzer, "Spatially heterogeneous dynamics in liquids: insight from simulation," Journal of Non-Crystalline Solids 274 (2000) 342-355.

[16] M. D. Ediger, "Spatially heterogeneous dynamics in supercooled liquids," Annu. Rev. Phys. Chem. 51 (2000) 99-128.

[17] P. K. Dixon and D. J. Durian, "Speckle visibility spectroscopy and variable granular fluidization," Physical Review Letters 90(18) (2003) 184302.

[18] P. A. Lemieux and D. J. Durian, "Investigating non-gaussian scattering processes by using n-th order intensity correlation functions," J. Opt. Soc. Am. A 16(7) (1999) $1651-1664$.

[19] P. A. Lemieux and D. J. Durian, "Quasi-elastic light scattering for intermittent dynamics," App. Opt. 40(24) (2001) 3984-3994.

[20] S. Bogdanovich and D. Popovic, "Onset of glassy dynamics in a two-dimensional electron system in silicon," Physical Review Letters 88(23) (2002) 236401-1.

[21] J. Jaroszynski, D. Popovic, and T. M. Klapwijk, "Universal behavior of the resistance noise across the metal-insulator transition in silicon inversion layers," Physical Review Letters 89(27) (2002) 276401-1.

[22] M. B. Weissman, "What is a spin-glass - a glimpse via mesoscopic noise," Reviews of Modern Physics 65(3) (1993) 829-839.

[23] L. Buisson, L. Bellon, and S. Ciliberto, "Intermittency in ageing," Journal of PhysicsCondensed Matter 15(11) (2003) S1163-S1179.

[24] L. Buisson, S. Ciliberto, and A. Garcimartin, "Intermittent origin of the large violations of the fluctuation-dissipation relations in an aging polymer glass," Europhysics Letters 63(4) (2003) 603-609.

[25] H. E. Castillo, C. Chamon, L. F. Cugliandolo, and M. P. Kennett, "Heterogeneous aging in spin glasses," Physical Review Letters 88(23) (2002) 237201.

[26] H. E. Castillo, C. Chamon, L. F. Cugliandolo, J. L. Iguain, and M. P. Kennett, "Spatially heterogeneous ages in glassy systems," Physical Review B 68(13) (2003) 134442.

[27] S. T. Bramwell, K. Christensen, J.-Y. Fortin, P. C. W. Holdsworth, H. Hensen, S. Lise, J. M. Lopez, M. Nicodemi, J.-F. Pinton, and M. Sellitto, "Universal fluctuations in correlated systems," Phys. Rev. Lett. 84(17) (2000) 3744-3747.

[28] N. Lacevic, F. W. Starr, T. B. Schroder, V. N. Norikov, and S. C. Glotzer, "Growing correlation length on cooloing below the onset of caging in a simulated glass-forming liquid," Phys. Rev. E 66 (2002) 030101. 